level of auto-antibodies and serum Ig did not significantly differ among the groups. However, crude mortality was significantly higher in mice treated with monoclonal anti-TNF compared to controls ( $p=0.0001$ for ADA and $p=0.0003$ for TN3) but not for mice treated with ETA (Figure). Incidence of lymphoma was higher in mice treated with monoclonal anti-TNF: $5 / 15$ (33\%) with TN3 ( $p=0.03 /$ controls), 4/12 (33\%) with ADA ( $p=0.054 /$ controls), $0 / 15$ with ETA and $1 / 22(5 \%)$ in controls.

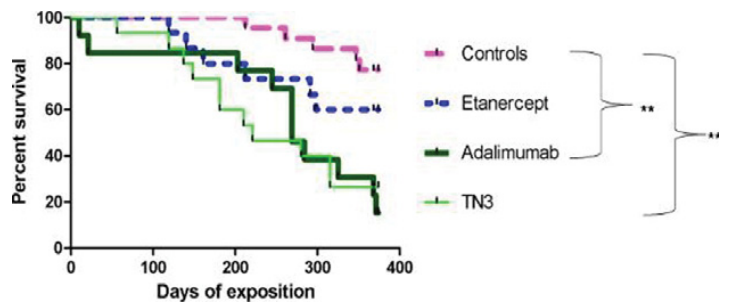

Conclusions: Higher mortality and increased risk of lymphoma were observed in BAFF Tg mice treated with monoclonal anti-TNF compared to etanercept. This result may be linked either to the different mechanism of action between the soluble receptor and the monoclonals or to a difference of trough level observed in the different groups even if higher levels of ADA was mandatory given the difference of effect on mouse TNF. This study demonstrates the negative impact of a prolonged anti-TNF treatment on the risk of lymphoma in the context of BAFF increase.

Disclosure of Interest: None declared

DOI: 10.1136/annrheumdis-2017-eular.5651

\section{OP0308 TNF INHIBITOR TREATMENT AND RISK OF CANCER RECURRENCE IN PATIENTS WITH RHEUMATOID ARTHRITIS: A NATIONWIDE COHORT STUDY FROM SWEDEN}

P. Raaschou, J. Söderling, J. Askling on behalf of the ARTIS study group. Dept of Medicine, Karolinska Institute, Stockholm, Sweden

Background: Clinical guidelines caution against the use of TNFi in individuals with a recent (5-10 years) history of cancer, but evidence of an increased risk of cancer recurrence is limited (1-2).

Objectives: We investigated the risk of recurrent solid non-skin cancer in patients with rheumatoid arthritis and TNFi-treatment, and if time between index-cancer and TNFi-start influence this risk.

Methods: 61.950 individuals with RA were identified in the Swedish national outpatient-care register Jan 2001-Dec 2014. Among these, 446 Individuals with at least one diagnosis of cancer (index cancer) prior to the start of TNFi-treatment were identified through linkage to the national cancer register and the ARTIS register of biologic treatment. Individuals $(n=1.278)$ with a history of equally recent cancer of the same type and stage (invasive/in situ) were matched 3:1 to each patient starting TNFi against a background of solid cancer. Study participants were required to be in cancer remission during a period of 6 months prior to start of follow-up. The primary outcome was first recurrence or second primary of the same cancer type, identified through register-linkages until Dec 2014. Hazard ratios (HR) for recurrence or second primary were calculated using a Cox regression model with TNFi-treatment start (and a corresponding date among the matched biologics-naïve individuals) as start of follow-up. The final models were stratified for the matching variables sex, birth year, year of diagnosis of the index cancer and index cancer type and stage (invasive vs in situ), and adjusted for education level and comorbidities.

Results: The mean time from index cancer diagnosis until TNFi-treatment/start of follow-up was 9.9 and 9.5 years among the TNFi treated and their matched biologics-naïve controls, respectively. The mean follow-up (SD) from TNFi start was 4.9 (3.5) and 4.1 (3.1) years, respectively. The cancer stage distribution was similar between the two groups, apart from stage IV $(0.6 \%$ among the TNFitreated and $1.6 \%$ among the biologics-naïve). Thirty individuals (7\%) among the 446 TNFi-treated developed a cancer recurrence (crude incidence rate 14/1000 person-years), compared with 89 (7\%) among the 1.278 matched biologics-naive (crude incidence rate 17/1000 person-years). This corresponded to an adjusted $\mathrm{HR}$ for recurrent cancer of $0.69(95 \% \mathrm{Cl} 0.42-1.12)$ in the matched analysis (table 1) comparing the TNFi treated to the biologics-naïve individuals. Stratified analyses indicated no increased risk associated with any specific cancer type with the possible exception of uterine cancer where HR for recurrence was 14.8 (95\% Cl 1.17-187.5), based on only 1 event among the TNFi-treated. HR for recurrence among individuals starting TNFi treatment within 5 year from index cancer was $0.67(0.31-1.44)$.

Conclusions: Among patients with RA and a history of cancer, those selected to receive TNFi-treatment in clinical practice did not experience more cancer recurrences than patients with RA treated otherwise. We detected no differential risk depending on the timing of TNFi-start in relation to the index cancer. The generalizability of our findings to individuals with a very recent cancer, or a poor prognosis, remains unknown.

[1] Raaschou P, et al. Ann Rheum Dis 2015;74:2137-2143.

[2] Mamtani R, et al. Arthritis Rheumatol 2016; 68: 2403-2411.
Table 1 Number of patients, recurrent or second primary cancer, incidence rate per 1000 personyears (PY) and hazard ratios (HR) among TNFi treated patients with RA and their matched biologics-naìve comparators

\begin{tabular}{|c|c|c|c|c|c|c|c|}
\hline \multirow[b]{2}{*}{ Cancer } & \multicolumn{3}{|c|}{ TNFi } & \multicolumn{3}{|c|}{ Matched comparators } & \multirow[b]{2}{*}{$\begin{array}{l}\text { Adjusted* HR } \\
\text { (95 \% CI) }\end{array}$} \\
\hline & $\mathbf{N}$ & Events (\%) & $\begin{array}{c}\text { IR } \\
\text { per } \\
1000 \\
\text { PY }\end{array}$ & $\mathbf{N}$ & Events (\%) & $\begin{array}{c}\text { IR } \\
\text { per } \\
1000 \\
\text { PY }\end{array}$ & \\
\hline Overall & 446 & $30(6.7 \%)$ & 13.8 & 1278 & $89(7 . \%)$ & 17.0 & $0.69(0.42-1.12)$ \\
\hline Anorectal & 29 & 0 & 0 & 69 & $4(5.8 \%)$ & 15.5 & - \\
\hline Breast & 212 & $22(10.4 \%)$ & 19.9 & 633 & $46(7.3 \%)$ & 16.1 & $1.04(0.57-1.89)$ \\
\hline CNS & 30 & $1(3.3 \%)$ & 7.0 & 81 & $3(3.7 \%)$ & 7.5 & - \\
\hline Colon & 34 & 0 & 0 & 91 & $3(3.3 \%)$ & 11.3 & $\cdot$ \\
\hline Kidney & 6 & 0 & 0 & 14 & $1(7.1 \%)$ & 16.4 & - \\
\hline Lung & 3 & $1(33.3 \%)$ & 86.1 & 9 & $(. \%)$ & 0 & - \\
\hline Ovarial & 29 & $1(3.4 \%)$ & 6.2 & 75 & $4(5.3 \%)$ & 12.8 & - \\
\hline Prostate & 59 & $2(3.4 \%)$ & 8.7 & 175 & $17(9.7 \%)$ & 31.0 & $0.16(0.03-1.00)$ \\
\hline Urinary & 16 & $2(12.5 \%)$ & 33.7 & 47 & $10(21.3 \%)$ & 65.5 & $0.80(0.16-4.07)$ \\
\hline Uterine & 28 & $1(3.6 \%)$ & 6.2 & 84 & $1(1.2 \%)$ & 2.8 & $=$ \\
\hline
\end{tabular}
Cox regression stratified for the matching variables sex, birth year ( \pm 10 years), year of diagnosis ( \pm 5 years) of the index cancer, cancer type and stage at diagnosis (invasive vs in situ) of the in therefore be calculated only when events occurred amonq both TNFi-treated and biologics-naive.

Disclosure of Interest: P. Raaschou: None declared, J. Söderling Grant/research support from: previous research projects fully or partly funded by Novo Nordisk and Combine Sweden, and has served as an external consultant to AbbVie, Merck and Novartis., J. Askling Grant/research support from: Abbvie, Pfizer, UCB, MSD, Roche, Lilly.

DOI: 10.1136/annrheumdis-2017-eular.5148

\section{FRIDAY, 16 JUNE 2017}

\section{AxSpA: from bug to gut and to disease phenotype -}

\section{OP0309 INTESTINAL SCLEROSTIN/SEROTONIN AXIS IS MODULATED BY DYSBIOSIS AND REGULATES ILC3 EXPANSION IN AS PATIENTS}

F. Ciccia $^{1}$, G. Guggino ${ }^{1}$, A. Rizzo ${ }^{1}$, S. Milling ${ }^{2}$, M. Luchetti $^{3}$, D. Baeten ${ }^{4}$, R. Alessandro ${ }^{1}$, G. Triolo ${ }^{1} .{ }^{1}$ University of Palermo, Palermo, Italy; ${ }^{2}$ University of Glasgow, Glasgow, United Kingdom; ${ }^{3}$ University Politecnica delle Marche, Ancona, Italy; ${ }^{4}$ University of Amsterdam, Amsterdam, Netherlands

Background: Sclerostin is an osteocyte-specific factor that binds to low-density lipoprotein receptor-related protein 5 (LRP5) inhibiting the Wnt signaling pathway and possibly contributing to the pathogenesis of Ankylosing spondylitis (AS). Subclinical gut inflammation observed in AS patients is characterized by the presence of dysbiosis and innate immune alterations. In the gut, LRP5 activation by unknown ligands inhibits serotonin production. Serotonin, by inducing glial derived neurotrophic factor (GDNF), controls ILC3 expansion, in the context of glial-ILC3-epithelial cell unit (GIECU). Sclerostin/serotonin axis has been never studied in AS.

Objectives: Aim of this study was to evaluate whether sclerostin is produced in the gut; to study the sclerostin/serotonin axis in AS and the effect of sclerostin on enterochromaffin cells $(E C)$; to evaluate the presence of intestinal GIECU in AS and the role of serotonin in modulating the production of GDNF on isolated intestinal glial derived cells. We finally studied the effect of GDNF on ILC3.

Methods: Ileal, synovial and bone marrow (BM) expression of sclerostin, serotonin and GDNF were investigated by rt-PCR, immunohistochemistry and WB in 30 AS patients and 20 controls. Platelet and plasma unconjugated concentrations of serotonin were assessed by high-performance liquid chromatography (HPLC). Isolated bacteria from AS ileal biopsies were cultured with EC and serotonin expression evaluated by RT-PCR. Sclerostin and serotonin gut expression were evaluated in HLA-B27 TG rats before and after antibiotics treatment. EC were stimulated with sclerostin and the expression of THP1 assessed by RT-PCR. The presence of GIECU was studied by confocal microscopy analysis of GFAP/Tbet/Thy1. Isolated intestinal glial cells were stimulated with serotonin and the modulation of GDNF assessed by RT-PCR. The effect of GDNF on ILC3 was evaluated by flow cytometry.

Results: Sclerostin was produced in the gut and down-regulated in AS. Upregulation of serotonin was observed in the gut, in the synovia and plasma, but not in BM of AS. Isolated intestinal bacteria from AS reduced EC serotonin production. Sclerostin down-regulation and serotonin over-expression were observed in the gut of HLA-B27 TG rats where Antibiotics increased intestinal sclerostin production and reduced serotonin expression. Treatment of isolated gut EC with sclerostin down-regulated the expression of THP1. GDNF was over-expressed in AS gut and confocal microscopy analysis demonstrated the existence of glial-ILC3-epithelial cells unit in AS patients. Finally, serotonin induces the release of GDNF by isolated intestinal glial cells and recombinant GDNF expanded RET+ILC3.

Conclusions: here we demonstrate for the first time that intestinal sclerostin is the ligand of LRP5 and modulates the release of serotonin. Sclerostin/serotin axis is dysregulated in AS patients and HLA-B27 TG rats. In HLA-B27 TG rats, antibiotics restored sclerostin production and serotonin expression indicating a role of dysbiosis in modulating sclerostin/serotonin axis. Serotonin seems to be an important regulator of ILC3 expansion by inducing the production of GDNF by enteric glial cells, in the context of glial-ILC3-epithelial cells unit.

Disclosure of Interest: None declared 